Surjono, H. \& Maltby, J. 2003. Adaptive Educational Hypermedia based on

Multiple Student Characteristics. In Proceedings of the Second International

Conference on Web-based Learning (ICWL 2003). Melbourne, Australia,

18-20 August 2003, CLNCS-Springer Verlag

\title{
Adaptive Educational Hypermedia based on Multiple Student Characteristics
}

\author{
Herman D. Surjono \\ Department of Electronic Engineering, Yogyakarta State University, Indonesia \\ hsurjo10escu. edu. au \\ John R. Maltby \\ School of Multimedia \& Information Technology, Southern Cross University \\ jmaltbyescu.edu.au
}

\begin{abstract}
The learning process in Adaptive Educational Hypermedia (AEH) environments is complex and may be influenced by aspects of the student, including prior knowledge, learning styles, experience and preferences. Current AEH environments, however, are limited to processing only a small number of student characteristics. This paper discusses the development of an AEH system which includes a student model that can simultaneously take into account multiple student characteristics. The student model will be developed to use stereotypes, overlays and perturbation techniques. Keywords: adaptive educational hypermedia, multiple characteristics, student model.
\end{abstract}

\section{Introduction}

The World Wide Web (WWW) has become a powerful environment for distributing information and many educational providers are using it to deliver knowledge to an increasingly wide and diverse audience. One of the initial approaches to delivering instruction via the Internet was to use web-based instruction (WBI). WBI is a hypermedia-based instructional program that utilizes the attributes and resources of the WWW to create a meaningful learning environment [1]. However, many WBIs are still oriented toward classroom style student groups that are expected to be homogeneous in terms of knowledge, motivation, learning styles, etc. Internet sites that use simple WBI tend to convey the same hypertext pages to every student, irrespective of the student's ability and background knowledge. This inflexibility provides many students with a less than optimum learning curve.

Students who study a course on the Internet tend to be more heterogeneously distributed than those found in a traditional classroom situation. Where this is the case, learning material should, if possible, be presented in a more personalised way. This problem is being addressed by the development of Adaptive Educational Hypermedia (AEH) systems. These systems combine ideas from hypermedia and intelligent tutoring systems to produce applications that adapt to meet individual educational needs. An AEH system dynamically collects 
and processes data about student goals, preferences and knowledge to adapt the material being delivered to the educational needs of the student [2]. Currently, however, most systems are capable of processing only a small number of student characteristics [3]. Since the learning process is influenced by many factors, including prior knowledge, experience, learning styles and preferences, it is important that the student model of an AEH system accommodates such factors in order to adapt accurately to student needs. This paper proposes the design of an AEH system with a student model that will simultaneously take into account multiple student characteristics.

\section{Adaptive Educational Hypermedia}

Adaptive hypermedia technology is actually a combination of two distinctive technologies, those of hypermedia and adaptive systems. According to Brusilovsky [2], adaptive hypermedia systems (AHS) can be defined as all hypertext and hypermedia systems that accommodate some user characteristics into the user model and apply this model to adapt various visible aspects of the system to the user. Three key components of the system are hypertext/hypermedia, the user model and the ability to adapt the hypermedia using the user model. According to De Bra [4], an AHS builds a user model by observing the user's browsing behaviour or by testing to determine what the user's background, experience, knowledge and interests are. These user characteristics are then used by the system to individualize the knowledge presentation. The presentation is adapted to the user model, and the user model is constantly updated as the user reads and interacts with the presentation.

Adaptive educational hypermedia (AEH) is one of the earliest applications of AHS and currently the most popular application in the field of adaptive systems. The advances of Internet technologies and the acceptance of distance learning have pushed many researchers and educators to develop educational hypermedia systems. Some interesting AEH that use the Web as a development platform include ELM-ART, InterBook, and 2L670. One of the most well-known general-purpose adaptive hypermedia systems is called AHA and it has been used to develop several educational adaptive hypermedia applications [5].

Two of the main advantages of Web-based instruction are classroom and platform independence: AEH extends these advantages by offering learners personalised instruction in a distance learning setting. Being adaptive is important when students have different needs, preferences, abilities, interests, behaviour, knowledge, etc. In addition, student knowledge and experience evolve over time in a student-dependent manner: students who have similar needs at the start of a course may follow very different paths and learning curves.

The basic components of existing AEH systems are the domain model, the user or student model and the adaptation model. The domain model is the subject area for which the adaptive hypermedia is intended as a resource, the student model is a collection of student characteristics and the adaptation model describes 
the parts of the hypermedia system that can be adapted and the circumstances under which this adaptation is to occur [6].

The main component of the AEH system is a student model that stores and processes relevant student characteristics. The student model maintains up-to-date information about each student's goals, background knowledge, etc. The system gathers information by asking the student to fill out questionnaires or achievement tests and by observing the browsing behavior of the student. The more precise and correct the student model, the more advanced the types of adaptation that can be supported.

In terms of method and technique for providing adaptation, Brusilovsky [7] identified two major areas: adaptive presentation (content adaptation) and adaptive navigation support (link adaptation). Adaptive presentation is the general term for techniques used to adapt the content of a web page based on the user model. These techniques include adaptive text presentation and adaptive multimedia presentation. Adaptive navigation support is the term for techniques used to modify the links accessible to the user at a particular time. These techniques include: direct guidance, adaptive link sorting, adaptive link hiding, adaptive link removal, adaptive link disabling, adaptive link annotation and map adaptation [2].

Most current systems are capable of considering only a small number of student characteristics for the adaptation [3]. According to Carver et al. [8] current student models normally limit characteristics to a single dimension. AES-CS [9] is an adaptive educational system that includes accommodations for cognitive styles in order to improve student interactions and learning outcomes. INSPIRE [10] is an adaptive system that monitors learner's activity and dynamically adapts the generated lessons to accommodate diversity in learner's knowledge state and learning style. NetCoach, an authoring system which supports the development of adaptive hypermedia, uses relations between visited pages and learner responses to test items [11]. AHA uses the fact that each student visits a specific page to update an estimate of his/her knowledge [12]. Whilst this is a practical approach, it has a major disadvantage: clearly, it is difficult to measure a student's knowledge simply by monitoring pages visited as it is not sure whether or not the student has really read and understood the information on each page.

\section{Student Model based on Multiple Student Characteristics}

The student model for the proposed AHS will be developed using techniques of stereotyping, overlays and perturbation. Stereotyping, where students are assigned to a specific group or stereotype, is one of the simplest of student modelling techniques. There are two types of stereotyping: fixed and default. In fixed stereotyping students are cast according to their performance into a predefined stereotype that is determined by academic level. Default stereotyping is a much more flexible approach: at the beginning of a session the students are stereotyped to default values, but as the learning process progresses and student 
performance data is obtained, the settings of the initial stereotype are gradually replaced by more individualized settings [13].

The overlay is a classic student modelling technique. An overlay model is a student modelling technique in which student performance is considered to be a subset of expert knowledge in the subject domain [14]. In order to use the overlay model, the domain knowledge must be able to be broken down into generic items such as rules, concepts, facts, etc. Basically, the model estimates the level of mastery of each item in the domain that is considered to be fully mastered by an expert. The perturbation model is an extension of the overlay model that represents the student's knowledge as including possible misconceptions as well as a subset of the expert's knowledge [15]. This extension allows for better remediation of student mistakes, since the fact that a student believes something that is incorrect is pedagogically significant.

In the construction of the student model, many different aspects can be taken into account, such as the existing student knowledge of the application domain, the cognitive and learning styles of the student, and the student goals, preferences and background. The student model will build a student profile that stores information for each student. Profile updating (in the case of overlay models), or an assignment of a student's characteristics to a profile (in the case of stereotype models), requires access to the information that the student gives to the system [16]. This information can be collected by querying the student or by observing his/her actions.

The learning process in an actual AEH environment is complex and influenced by many characteristics of the student. It is therefore important to consider accommodating as many of these characteristics as possible into the student model in order to generate an accurate adaptation. However, many student characteristics have been identified in the literature; it is therefore important to select for use in the student model only those characteristics that directly influence student achievement in the learning process, otherwise the design of the student model will become unnecessarily complex. The student characteristics to be considered in the proposed system are knowledge, learning styles, experience, background and preference. Other characteristics such as age, sex/gender, race/ethnicity, demographic data, interests, etc are not taken into account as they are considered significantly less influential to student achievement.

Information about student characteristics is generally initialised either with default values or by querying the student. Thereafter, it is maintained by the system and students may be able to review or edit their own profiles. Student actions and events at various conceptual levels, such as mouse clicks, task completion and requests for help, are reported by the application to the student profile as well. An analysis engine combines the student profile with other models of the system to derive new student information. The analysis engine can update the student profile with the derived information or initiate an action in the application. Constructing the student model, analysing a student profile and deriving new information can be done by using, amongst others, Bayesian techniques, logic-based techniques, machine learning techniques, stereotype-based techniques and inference rules [17]. 
Most of the existing AEH systems consider only student knowledge as a means for providing adaptivity. Student knowledge is a variable with a different time-dependent value for each student. Thus adaptive systems must evaluate student knowledge at appropriate stages throughout the learning process, recognise the changes in knowledge status and then update the student model accordingly. Assessment tests that are part of the educational material can include special questions to assess the learner's level of performance.

Typically, a student's learning style is initialised through the submission of a questionnaire the first time the student logs onto the system. This allows the learning style to be automatically determined and stored in an individual student model. Alternatively, the learning style can be directly initialised or updated by the student, who is offered the option to select his/her dominant learning style based on information provided by the system about the general characteristics of the different learning style categories.

Finally, information stored in the student model is a valuable resource which enables a tutor to monitor progress and study student attitude; in addition, a quantitative evaluation of learners' preferences, in terms of the time spent, performance, help request frequency, etc., can provide useful information about the quality of the material used.

\section{System Architecture}

The proposed system architecture is documented at high-level in Figures 1 and 2 using the UML notation. It consists of four major components: the interface, the student model (SM), the domain model (DM) and the adaptation model (AM). Students will interact with the system through the interface in the form of a web browser such as Microsoft Internet Explorer. The other modules of SM, DM, and AM will reside in a web server that supports a Java environment. The system architecture depicted in Figure 1 is composed from all components that are represented as packages, the standard UML grouping mechanism.

Stereotyping is initially used to store information whilst the system waits to gather something better; then the overlay method is employed. The conceptual model of the SM is shown in Figure 2.

The initial knowledge level of each student will be obtained from tests that are presented at the beginning of the first session. From the student responses collected by the system, the SM will assign a particular value to the student regarding his/her knowledge level. Students may choose not to do the initial test and in this case the SM will assign a default value to the student knowledge. Whilst the learning session is progressing the system will present an achievement test every time a student has completed a particular topic. The test score will update the student knowledge level in the SM. This knowledge level will then be used by the adaptation model for controlling the presentation of learning materials. 


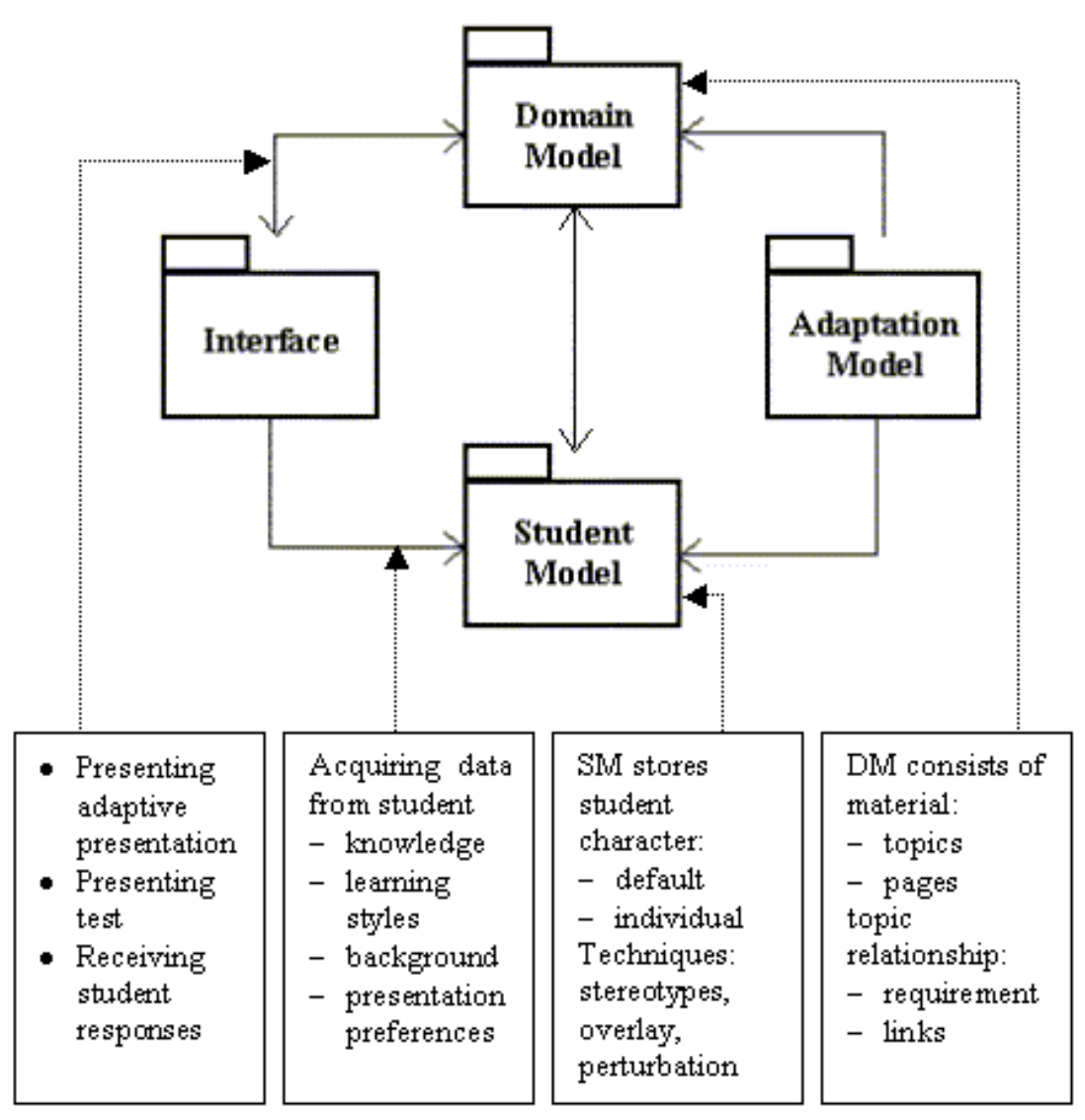

Fig. 1. The system architecture

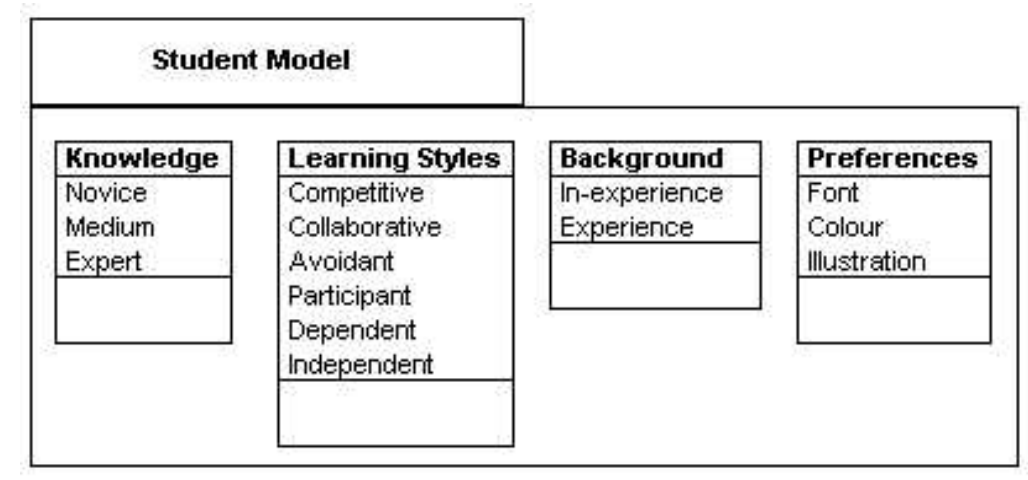

Fig. 2. The conceptual model of the student model

In order to learn optimally, especially in a distance learning environment, students must be aware of their own learning style. However, sometimes it is difficult for students to determine what their learning style is. The system will gather information about each student's style by presenting a questionnaire at the 
beginning of the learning session. There are many different categorizations of learning style inventories available in the literature. The Grasha-Riechmann Student Learning Styles Scale (GRSLSS) is ideal for assessing student learning styles in a college-level distance education setting [18]. The GRSLSS identifies six dimensions of learning styles as follows: competitive (compete with other students), collaborative (believe they can learn by sharing ideas), avoidant (uninterested by what happens in class), participant (eager to take part in class activities), dependent (need structure and support) and independent (like to work alone). Student scores for each of these dimensions will be stored in the SM so that they can influence the way the material is to be presented to the student.

The SM will also take into account the existing computing experience of the student. The system will offer selected questions to obtain an estimate of this experience. Students with little or no computing experience will receive special treatment until such time as their skill improves. The treatment will consist of presenting basic guidance on how to proceed, answer questions and do certain learning tasks; it will be employed only until a student has improved his/her skill to the necessary level. The final characteristics to be taken into account will be presentation preferences such as font, colour, illustration, etc.

The domain model (DM) contains a collection of learning materials. A course module implemented in the system can be divided into a number of topics with a particular value for each according to the level of difficulty. Each topic consists of several pages depending upon its complexity. The relationship between one topic and another can be expressed in terms of requirements such as pre-requisites, depending upon the nature of the topic. Students working on a particular topic can undertake tests at any time to evaluate their achievement for that topic. In order to advance to another topic which is at a higher academic level, students must pass a threshold score that is determined in the relationship rules.

The proposed AEH will be developed in the domain of electronics theory (a subject familiar to one of the authors) and targeted at university level students who are learning principles of electronics. Reasons why electronics is appropriate for use in web-based learning environments include the desirability of allowing students to practice and apply principal theories interactively and to provide immediate time-saving feedback to prevent students learning incorrect concepts. The uniqueness of this system compared to existing web-based learning systems for electronics theory is the capability to adapt the presentation to individual student needs. More specifically this system will take into account multiple student characteristics as a basis for providing the adaptation, so that the system will perform adaptation to students' needs more individually and accurately. This benefit is hoped to be the main contribution to the field of web-based learning systems.

The adaptation model (AM) contains adaptation and relationship rules. The adaptation rules describe how a page is presented to the student according to his/her own SM. Each time the student is assigned a score for a test, the SM will update his/her level of knowledge. In the proposed system, the level of knowledge will not be updated if the student is just accessing pages without undergoing a test; it is necessary to make sure that a student has understood a pre-requisite topic 
before advancing to a further topic. The relationship rules describe how any page or topic is related to other pages or topics in the DM.

The implementation of the AHS will use the following technologies. The student model and domain model will be implemented using XML/XSL files and a MySQL database. XML (Extensible Markup Language) and XSL (Extensible Stylesheet Language) technologies allow content to be separated from the presentation. XML is used to store the content and XSL is used to present pages with different layouts. The PHP scripting language will be used to present a dynamic content within a static document and is also appropriate for reading information from web forms and maintaining sessions between web pages. These sessions have to be maintained in order to store and update student names, browsing behaviour, student history and other student specific information in the student model. The adaptation model and interface will be implemented in a Java environment and enhanced by JavaScript and other graphical based software.

\section{Conclusion and Future Work}

Most existing AEH systems utilize student models that take account of only a small number of student characteristics. We contend that such models can provide only limited adaptability for the host application and, as such, do not optimize student learning for individual students. Since the learning process in an actual environment is complex and influenced by many aspects, it is important to consider these aspects in the design of an adaptive web-based learning system. Accordingly, research has commenced into prototyping an AEH system that incorporates a multi-dimensional student model to accommodate multiple student characteristics such as knowledge, learning styles, backgrounds and preferences.

\section{References}

1. Kahn, B.H., Web-Based Instruction (WBI): What is it and why is it?, In WebBased instruction, B.H. Kahn, Editor. 1997, Educational Technology Publications: Englewood Cliffs, NJ. p. 5-18.

2. Brusilovsky, P., Methods and techniques of adaptive hypermedia. User Modeling and User Adapted Interaction, 1996. 6(2-3): p. 87-129.

3. Carro, M.R. Adaptive Hypermedia in Education: New Considerations and Trends. In the 6th World Multiconference on Systemics, Cybernetics and Informatics. 2002. Orlando, Florida.

4. De Bra, P., Adaptive Educational Hypermedia on the Web. Communication of the ACM, 2002. 45(5): p. 60-61.

5. Calvi, L. and A. Cristea, Towards Generic Adaptive Systems: Analysis of a Case Study. Adaptive Hypermedia \& Adaptive Web-Based Systems, 2002. LNCS 2347: p. 79-89.

6. Pascoe, R. and A. Sallis. A Pedagogical Basis for Adaptive WWW Textbooks. In North American Web Developers Conference. 1998. 
7. Brusilovsky, P., Adaptive Hypermedia. User Modeling and User Adapted Interaction, 2001. 11: p. 87-110.

8. Carver, C.A.J., J.M.D. Hill, and U.W. Pooch. Third Generation Adaptive Hypermedia Systems. In World Conference on the WWW and Internet (WebNet 99). 1999. Honolulu, Hawaii.

9. Triantafillou, E., A. Pomportsis, and E. Georgiadou. AES-CS: Adaptive Educational System based on Cognitive Styles. In the Workshop on Adaptive System for Web-based Education, held in conjunction with AH'2002. 2002. Malaga, Spain.

10.Papanikolaou, K., A., et al. INSPIRE: An INtelligent System for Personalized Instruction in a Remote Environment. In Third Workshop on Adaptive Hypertext and Hypermedia. 2001. Berlin: Springer-Verlag.

11.Lippitsch, S., S. Weibelzahl, and G. Weber, Adaptive Learning Courses in Pedagogical Psychology The PSI Project and the authoring system NetCoach. Kunstliche Intelligenz, 2002. 3(02).

12.De Bra, P. and L. Calvi. AHA: a Generic Adaptive Hypermedia System. In the 2nd Workshop on Adaptive Hypertext and Hypermedia HYPERTEXT'98. 1998. Pittsburgh, USA.

13.Kay, J., Stereotypes, Student Models and Scrutability, In Proceedings of Fifth International Conference on Intelligent Tutoring Systems, G. Gauthier, C. Frasson, and K. VanLehn, Editors. 2000, Springer-Verlag: Montreal. p. 19-30.

14.Beck, J., M. Stern, and E. Haugsjaa, Applications of AI in Education, In ACM Crossroads Student Magazine. 1996.

15.Mayo, M.J., Bayesian Student Modelling and Decision-Theoretic Selection of Tutorial Actions in Intelligent Tutoring Systems, In Computer Science. 2001, University of Canterbury.

16.Cannataro, M., A. Cuzzocrea, and A. Pugliese. A Probabilistic Approach to Model Adaptive Hypermedia Systems. In 1st International Workshop on Web Dynamics (in Conjunction with the 8th International Conference on Database Theory). 2001. London, UK.

17.Kules, B., User Modeling for Adaptive and Adaptable Software Systems. 2000. [Available On-line] http://www.otal.umd.edu/UUGuide/wmk/, Accessed 17 December 2002.

18.Grasha, A.F., Teaching with styles: A practical guide to enhancing learning by understanding teaching and learning styles. 2002, Pittsburgh, PA: Alliance Publishers. 